\title{
A FORMULA FOR THE NUMBER OF PARTITIONS OF $n$ IN TERMS OF THE PARTIAL BELL POLYNOMIALS
}

\author{
SUMIT KUMAR JHA
}

\begin{abstract}
We derive a formula for $p(n)$ (the number of partitions of $n$ ) in terms of the partial Bell polynomials using Faà di Bruno's formula and Euler's pentagonal number theorem.
\end{abstract}

\section{MAIN REsUlt}

Recall the classical partition function, denoted by $p(n)$, gives the number of ways of writing the integer $n$ as a sum of positive integers, where the order of summands is not considered significant. For example, $p(4)=5$, since there are 5 ways to represent 4 as sum of positive integers, namely, $4=3+1=2+2=2+1+1=1+1+1+1$.

We also recall another classical statistic, the $(n, k)$ th partial Bell polynomial in the variables $x_{1}, x_{2}, \ldots, x_{n-k+1}$, denoted by $B_{n, k} \equiv \mathrm{B}_{n, k}\left(x_{1}, x_{2}, \ldots, x_{n-k+1}\right)$ ([1, p. 134], [5. Ch. 12]), defined by

$$
\mathrm{B}_{n, k}\left(x_{1}, x_{2}, \ldots, x_{n-k+1}\right)=\sum_{\substack{1 \leq i \leq n, \ell_{i} \in \mathbb{N} \\ \sum_{i=1}^{n} i \ell_{i}=n \\ \sum_{i=1}^{n} \ell_{i}=k}} \frac{n !}{\prod_{i=1}^{n-k+1} \ell_{i} !} \prod_{i=1}^{n-k+1}\left(\frac{x_{i}}{i !}\right)^{\ell_{i}} .
$$

Cvijović [3] gives the following formula for calculating these polynomials

$$
\begin{aligned}
B_{n, k+1}= & \frac{1}{(k+1) !} \underbrace{\sum_{\alpha_{1}=k}^{n-1} \sum_{\alpha_{2}=k-1}^{\alpha_{1}-1} \cdots \sum_{\alpha_{k}=1}^{\alpha_{k-1}-1}}_{k} \overbrace{\left(\begin{array}{c}
n \\
\alpha_{1}
\end{array}\right)\left(\begin{array}{c}
\alpha_{1} \\
\alpha_{2}
\end{array}\right) \ldots\left(\begin{array}{c}
\alpha_{k-1} \\
\alpha_{k}
\end{array}\right)}^{k} \times \cdots \\
& \times x_{n-\alpha_{1}} x_{\alpha_{1}-\alpha_{2}} \cdots x_{\alpha_{k-1}-\alpha_{k}} x_{\alpha_{k}} \quad(n \geq k+1, k=1,2, \ldots)
\end{aligned}
$$

We prove the following here.

Theorem 1. We have

$$
p(n)=\frac{1}{n !} \sum_{k=0}^{n}(-1)^{k} k ! B_{n, k}\left(\lambda_{1}, \lambda_{2}, \cdots, \lambda_{n-k+1}\right)
$$

where

$$
\lambda_{m}= \begin{cases}(-1)^{\frac{1+\sqrt{1+24 m}}{6}} m ! & \text { if } \frac{1+\sqrt{1+24 m}}{6} \in \mathbb{Z}, \\ (-1)^{\frac{1-\sqrt{1+24 m}}{6}} m ! & \text { if } \frac{1-\sqrt{1+24 m}}{6} \in \mathbb{Z}, \\ 0 & \text { otherwise. }\end{cases}
$$

Proof. We begin by the following generating function [2, Equation 22.13]

$$
\sum_{n \geq 0} p(n) q^{n}=\prod_{j=1}^{\infty} \frac{1}{1-q^{j}}
$$

2010 Mathematics Subject Classification. 05A17, 11P81.

Key words and phrases. Integer partitions, Partial Bell polynomials, Pentagonal numbers, Faà di Bruno's formula, Ramanujan's tau function. 
We recall the Euler's pentagonal number theorem [2, Equation 7.8]

$$
\begin{aligned}
E(q) & :=\prod_{j=1}^{\infty}\left(1-q^{j}\right)=\sum_{n=-\infty}^{\infty}(-1)^{n} q^{\frac{3 n^{2}+n}{2}} \\
& =1-q-q^{2}+q^{5}+q^{7}-q^{12}-q^{15}+q^{22}+q^{26}-\cdots .
\end{aligned}
$$

Let $f(q)=1 / q$. Using Faà di Bruno's formula ([1, p. 137], [5, Ch. 12]) we have

$$
\frac{d^{n}}{d q^{n}} f(E(q))=\sum_{k=0}^{n} f^{(k)}(E(q)) \cdot B_{n, k}\left(E^{\prime}(q), E^{\prime \prime}(q), \ldots, E^{(n-k+1)}(q)\right) .
$$

Since $f^{(k)}(q)=\frac{(-1)^{k} k !}{q^{k+1}}$ and $E(0)=1$, letting $q \rightarrow 0$ in the above equation gives

$$
p(n) n !=\sum_{k=0}^{n}(-1)^{k} k ! B_{n, k}\left(E^{\prime}(0), E^{\prime \prime}(0), \ldots, E^{(n-k+1)}(0)\right) .
$$

Then Euler's pentagonal number theorem (5) gives us

$$
E^{(m)}(0)=\lambda_{m}
$$

where $\lambda_{m}$ is as defined in (3).

Combining equations (1) and (2) we can conclude that

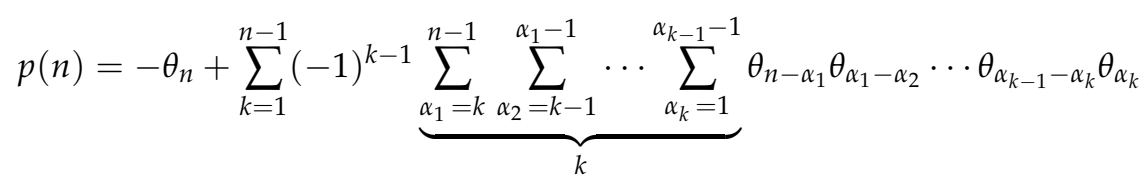

where

$$
\theta_{m}= \begin{cases}(-1)^{\frac{1+\sqrt{1+24 m}}{6}} & \text { if } \frac{1+\sqrt{1+24 m}}{6} \in \mathbb{Z}, \\ (-1)^{\frac{1-\sqrt{1+24 m}}{6}} & \text { if } \frac{1-\sqrt{1+24 m}}{6} \in \mathbb{Z}, \\ 0 & \text { otherwise. }\end{cases}
$$

Corollary 1. Let $E(q)^{r}:=\prod_{j=1}^{\infty}\left(1-q^{j}\right)^{r}=\sum_{n=0}^{\infty} p_{r}(n) q^{n}$ with $p_{r}(0)=1$ (see [4]). Then

$$
p(n)=\sum_{r=0}^{n}(-1)^{r}\left(\begin{array}{l}
n+1 \\
r+1
\end{array}\right) p_{r}(n),
$$

where by the virtue of the Faà di Bruno's formula (6) with $f(q)=q^{l}$ we have

$$
p_{l}(n)=\frac{1}{n !} \sum_{k=0}^{l}\left(\begin{array}{l}
l \\
k
\end{array}\right) k ! B_{n, k}\left(\lambda_{1}, \ldots, \lambda_{n-k+1}\right) .
$$

Proof. We start with the generating function for the partial Bell polynomials [1, Equation ( $\left.3 \mathrm{a}^{\prime}\right)$ on p. 133] as follows

$$
\begin{aligned}
\sum_{n=k}^{\infty} B_{n, k}\left(\lambda_{1}, \ldots, \lambda_{n-k+1}\right) \frac{q^{n}}{n !} & =\frac{1}{k !}\left(\sum_{j=1}^{\infty} \lambda_{j} \frac{q^{j}}{j !}\right)^{k} \\
& =\frac{1}{k !}(E(q)-1)^{k} \\
& =\frac{1}{k !} \sum_{r=0}^{k}(-1)^{k-r}\left(\begin{array}{l}
k \\
r
\end{array}\right) E(q)^{r} \\
& =\frac{1}{k !} \sum_{r=0}^{k}(-1)^{k-r}\left(\begin{array}{l}
k \\
r
\end{array}\right) \sum_{n=0}^{\infty} p_{r}(n) q^{n}
\end{aligned}
$$


to conclude that

$$
B_{n, k}\left(\lambda_{1}, \ldots, \lambda_{n-k+1}\right)=\frac{n !}{k !} \sum_{r=0}^{k}(-1)^{k-r}\left(\begin{array}{l}
k \\
r
\end{array}\right) p_{r}(n) .
$$

The above equation, together with the formula (2), gives us

$$
\begin{aligned}
p(n) & =\sum_{k=0}^{n} \sum_{r=0}^{k}(-1)^{r}\left(\begin{array}{l}
k \\
r
\end{array}\right) p_{r}(n) \\
& =\sum_{r=0}^{n}(-1)^{r} p_{r}(n) \sum_{k=r}^{n}\left(\begin{array}{l}
k \\
r
\end{array}\right) \\
& =\sum_{r=0}^{n}(-1)^{r}\left(\begin{array}{l}
n+1 \\
r+1
\end{array}\right) p_{r}(n) .
\end{aligned}
$$

Remark 1. Note that similar ideas are used in [6] with relation to partition zeta functions.

\section{REFERENCES}

[1] L. Comtet, Advanced Combinatorics: The Art of Finite and Infinite Expansions, D. Reidel Publishing Co., Dordrecht, 1974 .

[2] N. J. Fine, Basic Hypergeometric Series and Applications, American Mathematical Soc., 1988.

[3] D. Cvijović, New identities for the partial Bell polynomials, Applied mathematics letters, 24 (2011), 1544-1547.

[4] A. D. Forbes, Congruence properties of functions related to the partition function, Pacific J. Math. 158 (1993), 145156.

[5] G. E. Andrews, The Theory of Partitions, Cambridge University Press, 1988.

[6] K. Ono, L. Rolen, R. Schneider, Explorations in the Theory of Partition Zeta Functions, In: Exploring the Riemann Zeta Function. Springer, Cham, 2017.

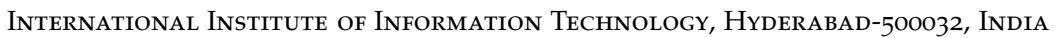

Email address: kumarjha.sumit@research.iiit.ac.in 